\title{
Ubiquity of the SARS-CoV-2 receptor ACE2 and upregulation in limbic regions of Alzheimer's disease brain
}

\author{
Yuhai Zhao ${ }^{1,2}$, Wenhong $\mathrm{Li}^{1,3}$, Walter J. Lukiw ${ }^{1,4,5}$ \\ ${ }^{1}$ LSU Neuroscience Center, Louisiana State University Health Sciences Center, New Orleans, LA, USA, ${ }^{2}$ Department of Anatomy \\ and Cell Biology, LSU Health Sciences Center, New Orleans, LA, USA, ${ }^{3}$ Department of Pharmacology, School of Pharmacy, Jiangxi \\ University of TCM, Nanchang, Jiangxi, China, ${ }^{4}$ Department of Ophthalmology, Louisiana State University Health Sciences Center, \\ New Orleans, LA, USA, ${ }^{5}$ Department of Neurology, Louisiana State University Health Sciences Center, New Orleans LA, USA
}

\begin{abstract}
The major route of entry for the severe acute respiratory syndrome coronavirus-2 (SARS-CoV-2) into human host cells is by means of the angiotensin-converting enzyme-2 (ACE2) transmembrane receptor. This zinc-containing carboxypeptidase and membrane-integral surface receptor is ubiquitous and widely expressed in multiple cell types. Hence SARSCoV-2, an unusually large RNA virus that causes coronavirus disease 2019 (COVID-19) has the remarkable capacity to invade many different types of human host cells simultaneously. Although COVID-19 is generally considered to be primarily an acute respiratory disease SARS-COV-2 also targets specific anatomical regions of the central nervous system (CNS). In the normal CNS the highest ACE2 levels of expression are found within the medullary respiratory centers of the brainstem and this, in part, may explain the susceptibility of numerous COVID-19 patients to severe respiratory distress. About 35\% of all COVID-19 patients experience neurological and neuropsychiatric symptoms, and a preexisting diagnosis of Alzheimer's disease (AD) predicts the highest risk of COVID-19 yet identified, with the highest mortality among elderly $A D$ patients. In the current study of multiple anatomical regions of $A D$ brains compared to age-, post-mortem interval- and gender-matched controls ( $n=10$ regions, $n=32$ brains), ACE2 expression was found to be significantly up-regulated in $A D$ in the occipital lobe, temporal lobe neocortex and hippocampal CA1. The temporal lobe and hippocampus of the brain are also targeted by the inflammatory neuropathology that accompanies $A D$, suggesting a significant mechanistic overlap between COVID-19 and AD, strongly centered on invasion by the neurotropic $S A R S$-COV-2 virus via the increased presence of ACE2 receptors in limbic regions of the AD-affected brain.
\end{abstract}

Key words: Alzheimer's disease, angiotensin-converting enzyme 2 (ACE2) receptor, Botzinger complex, COVID-19, coronavirus, SARS-CoV-2, single stranded RNA (sSRNA), TMPRSS2.

\section{Introduction}

The coronavirus disease pandemic, named COVID-19 by the World Health Organization (WHO), originated in late 2019 in an open air market in the city of Wuhan, Hubei Province in east-central China [1-3]. Initial reports suggested that the emergence of the novel SARS-CoV-2 virus, the causative agent of COVID-19, may have occurred via 'zoonotic spillover' from the fruit bat (Pteropus scapulatus), the Chinese

\section{Communicating author}

Walter J. Lukiw, MS, PhD, Prof. of Neuroscience, Neurology and Ophthalmology, Bollinger Professor of Alzheimer's disease (AD), LSU Neuroscience Center of Excellence, Louisiana State University Health Sciences Center, 2020 Gravier Street, Suite 904, New Orleans, LA 70112-2272 USA, e-mail: wlukiw@lsuhsc.edu 
pangolin (Manis pentadactyla) and/or other mammalian vectors through interspecies viral transmission $[1,10,13,21,25,32]$. To date over $\sim 162$ million cases of COVID-19 have been reported worldwide with over $\sim 3.4$ million deaths at the time of the writing of this report $[13,29]$.

Of COVID-19 patients and survivors many report an extremely wide array of respiratory, sensorial and neurological symptoms including intermittent loss of respiratory control, loss of taste (ageusia) and smell (anosmia), disruption in the coordination of movement (ataxia), loss of balance (vertigo), dizziness and light-headedness (presyncope), headache and nausea, direct negative effects of a heightened immune reaction and unusual hyper-inflammatory responses as a consequence of a prolonged 'cytokine storm', with indications of increased cardiovascular and neurovascular pathology and symptoms of an exacerbation or de novo induction of inflammatory neurodegeneration [2,3,34,36]. In addition there are widely reported progressive disturbances in cognition, anxiety and intermittent impairment or loss of consciousness, acceleration or aggravation of pre-existing cognitive deficits including a long-lasting 'brain fog' and a persistent and continuing disruption in understanding, reasoning and memory $[2,17,19,20,22,34-36]$. These deficits suggest the potential involvement of specific anatomical regions of the brain involved in perception, memory processing and cognition strikingly similar to those same brain areas targeted by Alzheimer's disease (AD). To cite 4 recent examples: (i) in the analysis of primary health records for over $\sim 13,300$ UK individuals tested for COVID-19 between March and July 2020 it was shown that a pre-existing diagnosis of AD predicted the highest risk of COVID-19 yet found, driving the highest mortality rate amongst any classification of aged individuals [16,33]; (ii) the morbidity and mortality of COVID-19 are elevated in $A D$ due to multiple pathological changes in $A D$ patients such as the excessive expression of the ACE-2 receptor and pro-inflammatory cytokines and chemokines, various ancillary complications of $A D$ including cardiovascular and neurovascular disease, diabetes, delirium and lifestyle alterations in AD [30]; (iii) multiple COVID-19 patients have reported various neurologic symptoms including AD-type cognitive impairment in part through COVID-19-induced inflammation, and post-COVID-19 syndrome [8]; and (iv) infection by SARS-CoV-2 both increases physiological and psychological stress and aggravates inflammatory reactions including the 'cytokine storm', supporting the deterioration of neuronal function and accelerating the progression of $A D[8,18]$.

Two previous reports of increased angiotensinconverting enzyme-2 (ACE2) expression in $A D$ have appeared in the literature, however they were both limited studies involving only one brain region and/ or post-mortem intervals (PMIs) of brain tissues $>13$ hours $[4,11]$. In the present report we have analyzed the protein levels of ACE2 and human transmembrane protease, serine 2 TMPRSS2, an ACE2 cellular serine protease that facilitates cellular SARSCoV-2 entry [23], in 10 brain regions from 32 control and AD brains with mean PMIs of 3.1-3.2 hours for all tissue samples analyzed. This is the first report of multiple regions of expression of the SARS-CoV-2 entry protein ACE2 and TMPRSS2 in limbic regions of the brain known to be involved in cognition and memory, which are the same anatomical regions targeted by the AD process. While TMPRSS2 protein showed no significant change in abundance between control and $A D$ when the same anatomical area was compared, ACE2 receptor protein abundance was found to be significantly elevated in the occipital pole (Brodmann area 17; the primary visual cortex), the temporal lobe neocortex (Brodmann area 22) and the hippocampal CA1 region. The latter two anatomical regions comprise a major part of the brain's limbic system normally involved in the processing of emotion, behavior, olfaction, cognition and memory.

\section{Material and methods \\ Human brain tissues}

Total protein was extracted from about $\sim 20 \mathrm{mg}$ (wet weight) samples of 9 control and 23 AD postmortem human brains; post-mortem human brain tissues of the highest quality available were obtained from 18 domestic and international brain banks (listed in the Acknowledgements section); the mean post-mortem interval (PMI; brain-death to brain-freezing at $\left.-81^{\circ} \mathrm{C}\right) \pm$ one standard deviation was $3.1 \pm 1.1$ hours for control and $3.3 \pm 1.4$ hours for $A D$; the mean age of the brain tissue donors was $76.3 \pm 12.4$ year for control and $78.1 \pm 11.7$ year for AD brain samples; there were no significant differences between the PMI, age or gender between the control and $A D$ groups. All tissues were from female donors. 


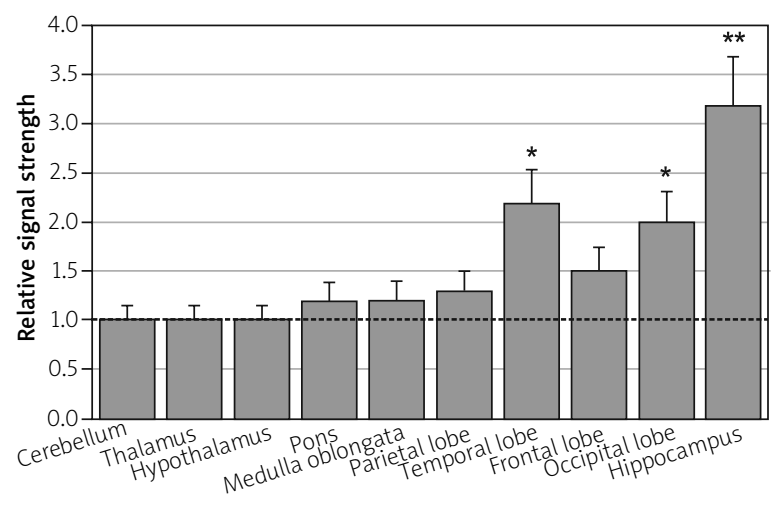

Fig. 1. ACE2 expression in different brain regions in Alzheimer's disease (AD). Tissue-specific patterns of ACE2 expression at the protein level in human brain cerebellum, thalamus, hypothalamus, pons, medulla oblongata, parietal lobe, temporal lobe association neocortex (Brodmann area A22), frontal lobe, occipital lobe and hippocampus (hippocampal CA1) were determined using a human ACE2 ELISA Kit (ab235649; Abcam, Cambridge MA, USA) in AD vs. age-, gender- and PMI-matched elderly controls; the control group $(n=9)$ had a mean age of $75.5 \pm 12.7$ years and a mean post-mortem interval (PMI; death to brain-freezing period) of $\sim 3.5$ hours; the AD group $(n=23)$ had a mean age of $76.1 \pm 11.4$ years and a mean post-mortem interval (PMI) of $\sim 3.4$ hours; all brain samples were from female donors; there was no significant difference in the mean age, gender, $\mathrm{PMI}$, yield or purity of total protein between the control and the $A D$ groups; in control human brain the pons, containing the medullary respiratory centers exhibits the highest concentration of ACE2 receptors of 21 brain regions analyzed $[13,17$; see text]; no significant difference in ACE2 receptor expression (at the level of protein) was found between control or AD thalamus and in this brain region the relative signal strength was set to 1.0; the pons was found to have an ACE2 expression of 1.15 AD over control which was not significant; on the other hand the occipital lobe, temporal lobe neocortex and hippocampus exhibited a 2-fold, 2.2-fold and 3.2-fold increases in ACE2 expression respectively; the temporal lobe association neocortex and hippocampus are part of the brain's limbic system and neuroanatomical regions targeted by the neuropathology of AD; all results are represented as relative signal strength which is defined as fold-change increases in $A D$ over control; a minimum of $n=3$ ELISA analyses were performed for each protein determination in tissues; ${ }^{*} p<0.05$, ${ }^{* *} p<0.01$ (ANOVA); error bars represent one standard deviation of the mean; a dashed horizontal line at 1.0 is included for ease of comparison; a preliminary report on ACE2 expression in AD compared to controls has recently been accepted for publication in the peer-reviewed literature: Lukiw WJ, SARS-CoV-2, the angiotensin converting enzyme 2 (ACE2) receptor and Alzheimer's disease. J Alzheimers Dis Parkinsonism 11: 520 (2021).
Ten brain regions including the cerebellum, thalamus, hypothalamus, pons, medulla oblongata, frontal lobe (Brodmann area 9), parietal lobe (Brodmann area 5), occipital lobe (Brodmann area 17; primary visual cortex), temporal lobe (Brodmann area 22; association neocortex) and the hippocampal CA1 region were extracted and processed as previously described [12]. The original CERAD (Center to establish a registry for Alzheimer's disease)/National Institutes of Health criteria were used to categorize $A D$ tissues in accordance with established guidelines [15]. All brain tissues were banked between 2015 and 2020 using the original CERAD criteria, and all of the AD tissues used in these studies had a clinical dementia rating (CDR) of 2.0-3.0, indicating the moderate- to severe stage of this progressive, age-related neurological disorder.

\section{ACE 2 and TMPRSS 2 protein analysis}

Protein extracts from control or AD tissues were generated using a ProteoExtract Complete Mammalian Proteome Extraction Kit (cat. no. 539779, Calbiochem/Millipore-Sigma Burlington MA) and were assayed for protein concentration using a Non-Interfering Protein Assay kit (cat. no. 488250, Calbiochem/Millipore-Sigma; quantified at $480 \mathrm{~nm}$ ); protein samples were stored in at $-81^{\circ} \mathrm{C}$ according to the manufacturer's protocol (Millipore-Sigma); ACE2 protein [UniProtKB - Q9BYF1 (ACE2_HUMAN)] [7,27] and TMPRSS2 protein [UniProtKB - 015393 (TMPS2 HUMAN)] $[27,28]$ abundance in these cell extracts of human brain regions were analyzed using a quantitative colorimetric $(450 \mathrm{~nm})$ sandwich ELISA specific for human ACE2 or TMPRSS2 employing a Fluoroskan Ascent FL Microplate Fluorometer and Luminometer (cat. no. 5200220, ThermoFisher Scientific, Waltham MA); we utilized a human ACE2 ELISA Kit ab235649; Abcam Cambridge MA, USA; sensitivity $1052 \mathrm{pg} / \mathrm{ml}$; detection range $1.5-255 \mathrm{ng} / \mathrm{ml}$ and a human (human transmembrane protease, serine 2 (TMPRSS2) ELISA Kit, cat. no. EKN48965 96T/ cat. no. EKU07854; Biomatik Wilmington DE; sensitivity $59 \mathrm{pg} / \mathrm{ml}$; detection range $0.156-10 \mathrm{ng} / \mathrm{ml}$ $[26,28]$. Human $\beta$-actin (anti- $\beta$-actin antibody (Abcam ab8227) were used as internal controls to quantify relative ACE2, TMPRSS 2 and $\beta$-actin protein abundance in each sample according to the manufacturer's instructions (Fig. 1); concentrations of ACE2, TMPRSS2 and $\beta$-actin were measured in tripli- 
cate and quantified according to the manufacturer's protocol (Supplementary File 1).

\section{Statistical analysis}

The analysis of statistical significance was evaluated using a two-way factorial analysis of variance ( $p$, ANOVA; SAS Institute, Cary NC, USA). A $p<0.05$ (ANOVA) was deemed as statistically significant; a $p<0.01$ (ANOVA) was deemed as very highly significant; experimental values were expressed as the means \pm one standard deviation (SD) of that mean (Fig. 1).

\section{Results}

Both ACE2 and TMPRSS2 proteins were abundantly detected in all 10 anatomical regions of the brain examined in this study. As determined by ELISA the mean abundance for the 805 amino acid $92.5 \mathrm{kDa}$ ACE2 protein was about $\sim 15 \mathrm{ng} / \mathrm{ml}$ extract and the mean abundance of TMPRSS2 protein was about $\sim 1.6 \mathrm{ng} / \mathrm{ml}$; in both cases $20 \mathrm{mg}$ wet weight of brain tissue were extracted using the protocol described above; the ACE2 receptor is therefore among the most abundant receptor types on the surface of human brain cells $[7,13,27]$. As quantified by ELISA there was no significant difference in the abundance of the 492 amino acid, $53.9 \mathrm{kDa}$ TMPRSS2 protein between control and $A D$ in any of these same 10 anatomical regions of the brain examined (Supplementary File 1). On the other hand ACE2 was significantly increased in the occipital lobe (Brodmann area 17; the primary visual cortex), temporal lobe (Brodmann area 22; association neocortex) and hippocampal CA1 to respectively 2.05 -fold, 2.25 -fold and 3.25-fold over age-, gender- and PMI-matched controls (Fig. 1). No significant changes for ACE abundance in the cerebellum, thalamus, hypothalamus, pons, medulla oblongata, frontal lobe (Brodmann area 9) or parietal lobe (Brodmann area 5) were noted in any of the AD tissues analyzed compared to controls $[13,16]$.

\section{Discussion}

The invasion of SARS-CoV-2 into human host cells occurs via the ACE2 receptor that is absolutely essential for viral infectivity and intracellular replication $[3,5,16,36]$. Accessory membrane enzymes such as the serine 2 transmembrane protease TMPRSS2 facilitates SARS-CoV-2 infection via two indepen- dent mechanisms, proteolytic cleavage of mono carboxypeptidase ACE2 at the intracellular C-terminal endodomain which promotes viral uptake, and cleavage of coronavirus spike (S1) glycoprotein which activates S1 for host cellular entry $[5,7,24]$. Besides enabling SARS-CoV-2 invasion, the ubiquitous ACE2 receptor is normally involved in the renin-angiotensin system (RAS), and functions in vasoconstriction and vasodilation, as a critical regulator of blood pressure, blood volume, systemic vascular resistance, amino acid trafficking and cardiovascular and neurovascular homeostasis [5,23,24,27]. The upregulation of ACE2, but not TMPRSS2, in anatomical regions involved in AD again underscores ACE2's importance as a key facilitator of SARS-CoV-2 infection in brain areas targeted for age-related neurodegeneration. The omnipresence of ACE2 expression in multiple human cell-, tissue-types and brain regions is noteworthy, and the elicitation of this SARS-CoV-2-mediated multipronged attack explains in part the severity and extensive variety of signs and symptoms widely observed in COVID-19 patients.

The SARS-CoV-2 virus has evolved a remarkable evolutionary strategy to ensure its replication, survival, proliferation and propagation by exploiting the presence of a very common, abundant, essential and susceptible transmembrane ACE2 receptor making many different neural and extra-neural human cell- and tissue-types at high risk and susceptibility to SARS-CoV-2 invasion. Another feature is that COVID-19 preferentially affects those patients with pre-existing medical conditions, such as a compromised immune and metabolic systems and those with neurological disorders that include AD. Besides strong ACE2 expression in respiratory epithelium, digestive, renal-excretory and reproductive cells, high ACE2 expression has also been found in the $A D$-affected limbic regions of the brain including the temporal association neocortex and hippocampal structures [13]. Elevated ACE2 levels in anatomical regions of the brain centrally involved in AD-type change suggests a significant mechanistic overlap between AD and COVID-19 strongly centered on invasion by the neurotropic SARS-CoV-2 via the ubiquity and overexpression of ACE2 receptors and their close association with inflammatory neurodegeneration. After the acute phase of COVID-19 infection and prospective recovery, the long-term neurological complications of SARS-CoV-2 neuroinvasion on the development of age-related neurodegenerative dis- 
orders are still not well understood [6,31]. COVID-19 is an acute condition while $A D$ is age-related and a disorder of the elderly, so it may be some time before the opportunity arises to examine SARS-CoV-2triggered neuropathological changes in a large number of post-mortem AD-affected human brains in order to obtain statistical strength for more robust correlation analysis $[6,16,17,31,33,35]$.

\section{Summary}

The current COVID-19 pandemic (i) has created an unparalleled global health crisis; and (ii) our scientific understanding of the mechanistic factors involved in SARS-CoV-2 transmission, invasion, viral spread and affinity for multiple human host cell targets continues to evolve. The ACE2 receptor, the gateway for SARS-CoV-2 entry into the host cell, has an extraordinary ubiquity and has been detected on the surface of all human cell types analyzed so far (with the exception of the erythrocyte) thus making it among the most prevalent receptor subtypes encountered in the human body. This indicates (i) that multiple cell types and tissues of the human respiratory, cardiovascular, digestive and genitourinary systems, hematic, lymphatic and glymphatic systems, and the central and peripheral nervous systems (CNS, PNS) provide many different potential entry portals for SARS-CoV-2 invasion; and (ii) this may in part explain the wide range of systemic involvement of SARS-CoV-2 infection and the variation in the many symptoms observed in both active and recovering COVID-19 patients. Abundant data further indicate that the high abundance of ACE2 in different brain cell types and neuroanatomical regions have a bearing on the widely reported incidence of disorientation, impairment of memory, reasoning and cognition and 'brain fog' not only during active SARS-CoV-2 disease but also during the often challenging period of recuperation following COVID-19 infection.

The following are related to these observations: (i) SARS-CoV-2 directly infects human neurons $[23,24]$; and (ii) the highest ACE2 receptor abundance in the normal CNS has been found in the pons and medulla oblongata of the lower human brainstem that contain the medullary respiratory regulatory rhythm generators, including the Botzinger complex that regulates inspiration during respiratory activity, and this in part may explain the susceptibility of numerous COVID-19 patients to severe respiratory distress $[13,33]$. Another remarkable finding is the neurotropism of SARS-CoV-2 towards highly specific brain regions involved in memory and cognition that are also targeted by the inflammatory neurodegeneration characteristic of that observed in $A D$ brain $[6,12]$. The highest density of the ACE2 receptors in the brain colocalize to areas also targeted by $A D$ including the occipital lobe containing the primary visual cortex and the temporal lobe and hippocampal CA1, key anatomical regions involved in visual perception and interpretation and the limbic system collectively involved in visual signal processing, cognition, behavior and memory formation [6,31]. It has been shown recently that a pre-existing diagnosis of $A D$ predicts the highest risk yet found for the development of COVID-19, and is further associated with the highest mortality rate among adults $>65$ years of age - these constitute a very high-risk elderly group prone to the most severe cases of SARS-CoV-2 often with a lethal outcome $[16,17,31]$. Our current healthcare system could be faced with a significantly increased volume of AD patients dealing with these and other associated issues of neurological comorbidity in older adults after the most severe cases of SARS-CoV-2 infection, requiring long-term neurological follow-up in the often difficult aftermath of COVID-19 recovery.

\section{Acknowledgements}

The experimental, analytical and statistical work in this communication was presented in part at the Society for Neuroscience (SFN) online Annual Meeting on 19-23 October 2019 in Chicago IL, USA. Sincere thanks are extended to the late Drs. JM Hill (JMH; Louisiana State University) for helpful discussions on this research area and to F. Culicchia, C. Eicken, C. Hebel, B. Krishnan, K. Navel, and L. Wong for short postmortem interval (PMI) human and other mammalian brain tissues or extracts and to D. Guillot and A.I. Pogue for expert technical assistance. Thanks are also extended to the many neuropathologists, physicians and researchers of the US, Canada, Europe and the Russian Federation who have provided high quality, short postmortem interval (PMI) human CNS or extracted brain tissue fractions for scientific study. We would like to further thank the following 18 domestic and international brain banks, and their continuing cooperation, for access to high quality short PMI tissues and valuable analytical advice: the Autism Brain Net, Los Angeles, 
CA, USA; the Harvard University/McLean Hospital Tissue Center, Boston, MA, USA; Louisiana State University, New Orleans, LA, USA; the Lomonosov Institute, Moscow State University, Moscow, Russian Federation; the National Disease Research Interchange, Philadelphia, PA, USA; the National Institutes of Health NIH NeuroBioBank, comprised of tissues from the National Institute of Mental Health (NIMH), the Eunice Kennedy Shriver National Institute of Child Health and Human Development (NICHD), and the National Institute of Neurological Disorders and Stroke (NINDS), Bethesda, MD, USA; the Netherlands Brain Research Institute, Amsterdam, Netherlands; the New York State Institute for Basic Research, Staten Island, NY, USA; the Oregon Health Sciences University, Portland, OR, USA; the Southern Eye Bank, Metairie, LA, USA; the University of California, Irvine, CA, USA; the University of Kentucky Alzheimer's disease Brain Bank, Lexington, KY, USA; the University of Maryland Brain and Tissue Bank, Baltimore, MD, USA; the University of Massachusetts, Worcester, MA, USA; University of Pennsylvania School of Medicine, Philadelphia, PA, USA, and the University of Toronto Brain Bank, Toronto, ON, Canada. All authors agree on the content of this publication. Research on metal neurotoxicity, human and murine microRNAs, small noncoding RNA (sncRNA), proinflammatory and pathogenic signaling in the Lukiw laboratory involving the innate-immune response, amyloidogenesis and inflammatory neurodegeneration in AD, ASD, PrD and in other human neurological disorders was supported through an unrestricted grant to the LSU Eye Center from Research to Prevent Blindness (RPB); the Louisiana Biotechnology Research Network (LBRN), the Alzheimer Association and NIH grants NEI EY006311, NIA AG18031 and NIA AG038834 (WJL).

\section{Ethics statement}

All acquisition, handling, experimental and analytical procedures involving postmortem human brain tissues were carried out in an ethical manner in strict accordance with the ethics review board policies at brain and tissue donor institutions and at the Louisiana State University (LSU) Health Sciences Center. Informed consent from next of kin was obtained at brain and tissue donor institutions for all tissue samples prior to autopsy and donation; coded postmortem brain tissue samples (containing no personal identifying information of the donors) were obtained from the 18 brain and tissue banks listed in the Acknowledgements section above. The ethical use of postmortem human brain tissues and their analyses were also carried out in strict accordance with the Institutional Biosafety Committee and the Institutional Review Board Committee (IBC/ IRBC) ethical guidelines IBC\#18059 and IRBC\#6774 at the LSU Health Sciences Center, New Orleans, LA 70112, USA.

\section{Disclosure}

The authors report no conflict of interest.

The experimental work in this paper was funded by the LSU Eye Center from Research to Prevent Blindness (RPB), the Louisiana Biotechnology Research Network (LBRN), the National Institutes of Health $(\mathrm{NIH})$, Bethesda, MD, USA and the Alzheimer Association Chicago, IL, USA.

The supplementary file 1 is available on journal website.

\section{References}

1. Adhikari SP, Meng S, Wu YJ, Mao YP, Ye RX, Wang QZ, Sun C, Sylvia S, Rozelle S, Raat H, Zhou H. Epidemiology, causes, clinical manifestation and diagnosis, prevention and control of coronavirus disease (COVID-19) during the early outbreak period: a scoping review. Infect Dis Poverty 2020; 9: 29

2. Amruta N, Chastain WH, Paz M, Solch RJ, Murray-Brown IC, Befeler JB, Gressett TE, Longo MT, Engler-Chiurazzi EB, Bix G. SARS-CoV-2 mediated neuroinflammation and the impact of COVID-19 in neurological disorders. Cytokine Growth Factor Rev 2021; 58: 1-15.

3. Baig AM, Sanders EC. Potential neuroinvasive pathways of SARS-CoV-2: deciphering the spectrum of neurological deficit seen in coronavirus disease 2019 (COVID-19). J Med Virol 2020; 92: 1845-1857.

4. Ding Q, Shults NV, Gychka SG, Harris BT, Suzuki YJ. Protein expression of angiotensin-converting enzyme 2 (ACE2) is upregulated in brains with Alzheimer's disease. Int J Mol Sci 2021; 22: 1687.

5. Gheblawi M, Wang K, Viveiros A, Nguyen Q, Zhong JC, Turner AJ, Raizada MK, Grant MB, Oudit GY. Angiotensin-converting enzyme 2: SARS-CoV-2 receptor and regulator of the reninangiotensin system: celebrating the 20th anniversary of the discovery of ACE2. Circ Res 2020; 126: 1456-1474.

6. Hascup ER, Hascup KN. Does SARS-CoV-2 infection cause chronic neurological complications? Geroscience 2020; 42: 1083-1087.

7. Hoffmann M, Kleine-Weber H, Schroeder S, Krüger N, Herrler T, Erichsen S, Schiergens TS, Herrler G, Wu NH, Nitsche A, Müller MA, Drosten C, Pöhlmann S. SARS-CoV-2 cell entry depends on ACE2 
and TMPRSS2 and is blocked by a clinically proven protease inhibitor. Cell 2020; 181: 271-280.e8.

8. Hu C, Chen C, Dong XP. Impact of COVID-19 pandemic on patients with neurodegenerative diseases. Front Aging Neurosci 2021; 13: 664965.

9. Ikeda K, Kawakami K, Onimaru H, Okada Y, Yokota S, Koshiya N, Oku Y, lizuka M, Koizumi H. The respiratory control mechanisms in the brainstem and spinal cord: integrative views of the neuroanatomy and neurophysiology. J Physiol Sci 2017; 67: 45-62.

10. Li MY, Li L, Zhang Y, Wang XS. Expression of the SARS-CoV-2 cell receptor gene ACE2 in a wide variety of human tissues. Infect Dis Poverty 2020; 9: 45.

11. Lim KH, Yang S, Kim SH, Joo JY. Elevation of ACE2 as a SARS CoV-2 entry receptor gene expression in Alzheimer's disease. J Infect 2020; 81: e33-e34.

12. Lukiw WJ, Zhao Y, Cui JG. An NF-kappaB-sensitive micro RNA-146a-mediated inflammatory circuit in Alzheimer disease and in stressed human brain cells. J Biol Chem 2008; 283: 31315-31322.

13. Lukiw WJ, Pogue A, Hill JM. SARS-CoV-2 infectivity and neurological targets in the brain. Cell Mol Neurobiol 2020; 1-8.

14. Mirzaei R, Mahdavi F, Badrzadeh F, Hosseini-Fard SR, Heidary M, Jeda AS, Mohammadi T, Roshani M, Yousefimashouf R, Keyvani H, Darvishmotevalli M, Sani MZ, Karampoor S. The emerging role of microRNAs in the severe acute respiratory syndrome coronavirus 2 (SARS-CoV-2) infection. Int Immunopharmacol 2021; 90: 107204.

15. Morris JC, Heyman A, Mohs RC, Hughes JP, van Belle G, Fillenbaum G, Mellits ED, Clark C. The consortium to establish a registry for Alzheimer's disease (CERAD). Part I. Clinical and neuropsychological assessment of Alzheimer's disease. Neurology 1989; 39: 1159-1165.

16. Nagu P, Parashar A, Behl T, Mehta V. CNS implications of COVID-19: a comprehensive review. Rev Neurosci 2020; 32: 219-234.

17. Uversky VN, Elrashdy F, Aljadawi A, Ali SM, Khan RH, Redwan EM. Severe acute respiratory syndrome coronavirus 2 infection reaches the human nervous system: How? J Neurosci Res 2021; 99: 750-777.

18. Pacheco-Herrero M, Soto-Rojas LO, Harrington CR, Flores-Martinez YM, Villegas-Rojas MM, León-Aguilar AM, MartínezGómez PA, Campa-Córdoba BB, Apátiga-Pérez R, Corniel-Taveras CN, Dominguez-García JJ, Blanco-Alvarez VM, Luna-Muñoz J. Elucidating the neuropathologic mechanisms of SARS-CoV-2 infection. Front Neurol 2021; 12: 660087.

19. Padda I, Khehra N, Jaferi U, Parmar MS. The neurological complexities and prognosis of COVID-19. SN Compr Clin Med 2020; 1-12.

20. Rahman MA, Islam K, Rahman S, Alamin M. Neurobiochemical cross-talk between COVID-19 and Alzheimer's disease. Mol Neurobiol 2021; 58: 1017-1023.

21. Rodriguez-Morales AJ, Bonilla-Aldana DK, Balbin-Ramon GJ, Rabaan AA, Sah R, Paniz-Mondolfi A, Pagliano P, Esposito S. History is repeating itself: Probable zoonotic spillover as the cause of the 2019 novel coronavirus epidemic. Infez Med 2020; $28: 3-5$.
22. Roy D, Ghosh R, Dubey S, Dubey MJ, Benito-León J, Kanti Ray B. Neurological and neuropsychiatric impacts of COVID-19 pandemic. Can J Neurol Sci 2021; 48: 9-24.

23. Samavati L, Uhal BD. ACE2, much more than just a receptor for SARS-COV-2. Front Cell Infect Microbiol 2020; 10: 317.

24. Song E, Zhang C, Israelow B, Lu-Culligan A, Prado AV, Skriabine S, LuP, Weizman OE, Liu F, Dai Y, Szigeti-Buck K, Yasumoto Y, Wang G, Castaldi C, Heltke J, Ng E, Wheeler J, Alfajaro MM, Levavasseur E, Fontes B, Ravindra NG, Van Dijk D, Mane S, Gunel M, Ring A, Kazmi SAJ, Zhang K, Wilen CB, Horvath TL, PluI, Haik S, Thomas JL, Louvi A, Farhadian SF, Huttner A, Seilhean D, Renier N, Bilguvar K, Iwasaki A. Neuroinvasion of SARS-CoV-2 in human and mouse brain. J Exp Med 2021; 218: e20202135.

25. Tiwari R, Dhama K, Sharun K, Iqbal Yatoo M, Malik YS, Singh R, Michalak I, Sah R, Bonilla-Aldana DK, Rodriguez-Morales AJ. COVID-19: animals, veterinary and zoonotic links. Vet Q 2020; 40: 169-182.

26. www.biomatik.com/elisa-kits/human-transmembrane-protease-serine-2-tmprss2-elisa-kit-cat-ekn07854/; last accessed 23 August 2021.

27. www.uniprot.org/uniprot/Q9BYF1; ACE2 - Angiotensin-converting enzyme 2 precursor - Homo sapiens (Human) - ACE2 gene \& protein (uniprot.org) UniProtKB - Q9BYF1 (ACE2 HUMAN); last accessed 23 August 2021.

28. www.uniprot.org/uniprot/O15393; TMPRSS2 - Transmembrane protease serine 2 precursor - Homo sapiens (Human) - TMPRSS2 gene \& protein (uniprot.org); last accessed 23 August 2021.

29. www.ourworldindata.org/covid-cases; https//ourworldindata. org/covid-deaths; last accessed 23 August 2021.

30. Xia X, Wang Y, Zheng J. COVID-19 and Alzheimer's disease: how one crisis worsens the other. Transl Neurodegener 2021; 10: 15.

31. Yachou Y, El Idrissi A, Belapasov V, Ait Benali S. Neuroinvasion, neurotropic, and neuroinflammatory events of SARS-CoV-2: understanding the neurological manifestations in COVID-19 patients. Neurol Sci 2020; 41: 2657-2669.

32. Ye ZW, Yuan S, Yuen KS, Fung SY, Chan CP, Jin DY. Zoonotic origins of human coronaviruses. Int J Biol Sci 2020; 16: 16861697.

33. Yu Y, Travaglio M, Popovic R, Leal NS, Martins LM. Alzheimer's and Parkinson's diseases predict different COVID-19 outcomes: a UK biobank study. Geriatrics (Basel) 2021; 6: 10.

34. Zhang Q, Shan KS, Abdollahi S, Nace T. Anosmia and ageusia as the only indicators of coronavirus disease 2019 (COVID-19). Cureus 2020; 12: e7918.

35. Zhou Z, Kang H, Li S, Zhao X. Understanding the neurotropic characteristics of SARS-CoV-2: from neurological manifestations of COVID-19 to potential neurotropic mechanisms. J Neurol 2020; 267: 2179-2184.

36. Zubair AS, McAlpine LS, Gardin T, Farhadian S, Kuruvilla DE, Spudich S. Neuropathogenesis and neurologic manifestations of the coronaviruses in the age of coronavirus disease 2019: a review. JAMA Neurol 2020; 77: 1018-1027. 